\title{
Polarized Neutron Diffraction to Probe Local Magnetic Anisotropy of a Low-Spin Fe(III) Complex
}

\author{
Karl Ridier, ${ }^{[a]}$ Abhishake Mondal, ${ }^{[b]}$ Corentin Boilleau, ${ }^{[c]}$ Olivier Cador, ${ }^{[c]}$ Béatrice Gillon, ${ }^{* a]}$ Grégory \\ Chaboussant, ${ }^{[a]}$ Boris Le Guennic, ${ }^{*[c]}$ Karine Costuas, ${ }^{[c]}$ Rodrigue Lescouëzec ${ }^{*[b]}$
}

\begin{abstract}
We have determined by PND the low temperature molecular magnetic susceptibility tensor of the anisotropic low-spin complex $\mathrm{PPh}_{4}\left[\mathrm{Fe}{ }^{\prime \prime \prime}(\mathrm{Tp})(\mathrm{CN})_{3}\right] \mathrm{H}_{2} \mathrm{O}$. We found the existence of a pronounced molecular easy magnetization axis, almost parallel to the $C_{3}$ pseudo-axis of the molecule, which also corresponds to a trigonal elongation direction of the octahedral coordination sphere of the Fe $\mathrm{el}^{\prime \prime \prime}$ ion. The PND results are coherent with Electron Paramagnetic Resonance (EPR), SQUID magnetometry and ab initio investigations. Through this particular example, we demonstrate the capabilities of PND to provide a unique, direct and straightforward picture of the magnetic anisotropy and susceptibility tensors, offering a clear-cut way to establish magneto-structural correlations in paramagnetic molecular complexes.
\end{abstract}

The understanding of the magnetic properties at the atomic scale is crucial to design molecule-based magnetic materials with original properties. There are only a few experimental techniques that enable to probe the magnetism at the local scale.

EPR provides information about the $g$ tensors and hyperfine constants. ${ }^{[1]}$ X-ray Magnetic Circular Dichroism (XMCD) gives access to local magnetic moments. ${ }^{[2]} \mathrm{NMR}$ allows to probe small amount of spin density in paramagnetic species. ${ }^{[3]}$ PND has proven to be one of the most efficient tools to enlighten the magnetic interaction mechanisms in molecule-based materials. ${ }^{[4]}$ In the present study, we show that PND can also be used to access the local magnetic anisotropy in molecular compounds. Actually, the prediction and the control of the magnetic anisotropy have become crucial for the design of advanced magnetic materials. For instance, the magnetic bistability in lowdimensional systems such as Single Molecule Magnets (SMMs) and Single Chain Magnets (SCMs) is critically linked to the anisotropy. ${ }^{[5]}$ The magnetic anisotropy is also of considerable importance in the design of nanodevices or nanostructured materials that show promises in spintronics and for data storage at the nanoscale. ${ }^{[6]}$

In this seminal study we have selected the mononuclear lowspin (LS) fac-[Fe $\left.\mathrm{Fe}^{\prime \prime \prime}(\mathrm{L})(\mathrm{CN})_{3}\right]^{-}$building block ( $\mathrm{L}$ is a ligand of the Tp family, with $\mathrm{Tp}=$ tris(pyrazolylborate) which has been widely

[a] Dr K. Ridier, Dr B. Gillon, Dr G. Chaboussant Laboratoire Léon Brillouin

CEA-CNRS, UMR12

CEA Saclay, 91191 Gif-sur-Yvette, France

E-mail: beatrice.gillon@cea.fr

[b] Dr A. Mondal, Prof. R. Lescouëzec

Institut Parisien de Chimie Moléculaire (IPCM),

Sorbonne Universités, UPMC Paris 6, UMR 8232

4 place Jussieu, 75252 Paris cedex 5, France

[c] Dr C. Boilleau, Dr O. Cador, Dr B. Le Guennic, Dr K. Costuas Institut des Sciences Chimiques de Rennes,

Université de Rennes 1, UMR 6226 CNRS

263 Av. du Général Leclerc, 35042 Cedex Rennes, France

Supporting information for this article is given via a link at the end of the document. used to synthesize functional magnetic materials. ${ }^{[7]}$ This complex, which exhibits a $S=1 / 2$ spin ground state, with a first order orbital magnetic momentum and a distorted octahedral symmetry should display a significant magnetic anisotropy. In this work we show how PND can give a direct picture of the local magnetic anisotropy, better than any other techniques, and enables to relate it to the molecular structure.

In the past decades, PND was largely used to study spin density distribution in paramagnetic molecular crystals $^{[8]}$ but most studies were restricted to the simpler case where all magnetic moments are aligned by the external magnetic field. In the presence of strong atomic anisotropy, the local field-induced moments are generally not collinear to the applied magnetic field and the paramagnetic analysis is no more valid. However, the diffraction process of polarized neutrons by a crystal is sensitive to the real orientation of the local magnetic moments in the crystal and many efforts have been recently devoted to the analysis of PND data in highly anisotropic paramagnets. ${ }^{[9]}$ One innovative method is based on the definition of a local susceptibility tensor $\overleftrightarrow{\chi}$ for each magnetic atom in the unit cell. ${ }^{[10]}$ The local magnetic moment $\boldsymbol{m}$ is related to the applied field $\boldsymbol{H}$ through the following relation (as long as the applied field is moderate enough to stay in the linear regime):

$$
\boldsymbol{m}=\overleftrightarrow{\chi} \boldsymbol{H}
$$

where $\overleftrightarrow{\chi}$ is a second rank symmetric tensor. The experimental quantities measured in a PND experiment are the flipping ratios $R(\boldsymbol{Q})$ defined as the ratio (for Bragg reflections of the scattering vector $\boldsymbol{Q})$ of the diffracted intensities for $(+)$ and $(-)$ polarized incident neutron beam. These experimental quantities contain information on both the orientation and the magnitude of the atomic magnetic moments throughout the unit cell and can be expressed in terms of susceptibility components $\chi_{i j}$.

The number of independent tensor components to be refined is determined by the local symmetry of the atomic site. In the most general case (local triclinic symmetry), the simultaneous refinement of the six independent tensor components $\chi_{i j}$ requires the measurement of at least three sets of flipping ratios. Those must be collected for three orthogonal directions provided that the magnetic response to the field is linear i.e. for sufficiently low magnetic fields. This method has already been employed to address the magnetic anisotropy in inorganic compounds. ${ }^{[11,12]}$ It has also been applied to molecular solids ${ }^{[13,14]}$ but only using high magnetic fields $(7 \mathrm{~T})$ for which the magnetization deviates strongly from linearity. In such cases, the tensor components were used as intermediate parameters for the determination of the local induced magnetic moments. In contrast, in the present study measurements were performed at low field, i.e. in the linear regime of magnetization, in order to access the genuine local susceptibility tensor and characterize the local magnetic anisotropy.

The crystal structure (monoclinic P2 $1 / \mathrm{c}$ ) of the $\mathrm{PPh}_{4}\left[\mathrm{Fe} e^{\text {III }}(\mathrm{Tp})(\mathrm{CN})_{3}\right] \cdot \mathrm{H}_{2} \mathrm{O}$ compound exhibits two independent complexes in the unit cell, ${ }^{[15]}$ with different orientations, thus 
hindering the access to the local magnetic anisotropy using usual macroscopic techniques. As a matter of fact, the macroscopic magnetic anisotropy, or "crystal anisotropy", arises from the combined effect of the intrinsic "molecular anisotropy" and the crystal symmetry i.e. the relative orientation of the individual molecules. In general the "crystal anisotropy" does not correspond to the "molecular anisotropy". They coincide if there is only one independent molecule in the lattice cell and if the molecular symmetry corresponds to the highest-order symmetry of the space group. Therefore, for numerous molecular compounds, macroscopic measurements performed on singlecrystal do not enable to access the molecular magnetic anisotropy. For example, cantilever torque magnetometry on single crystal gives a direct access to the molecular magnetic anisotropy only if iso-oriented molecules are present in the unit cell. ${ }^{[16]}$ This is not the case of PND, which allows to reveal the molecular anisotropy and to draw useful magneto-structural correlations.

The molecular structure of the $\left[\mathrm{Fe}^{\mathrm{III}}(\mathrm{Tp})(\mathrm{CN})_{3}\right]^{-}$anion is displayed in Fig. 1. The LS Fe ${ }^{\text {III }}$ ion is surrounded by three cyanide ligands in facial arrangement and a tridentate trispyrazolylborate (also referred as $\mathrm{Tp}^{-}$) leading to a distorted octahedral environment. The complex is close to the $\mathrm{C}_{3 v}$ symmetry and the $\mathrm{C}_{3}$ pseudoaxis passes through the $\mathrm{Fe}$ ion and the $\mathrm{B}$ atom of the $\mathrm{Tp}^{-}$ligand. Our (non-polarized) neutron diffraction data at $10 \mathrm{~K}$ (see SI) show the existence of a trigonal elongation of the $\mathrm{Fe}^{\text {III }}$ coordination environment along the molecular $\mathrm{C}_{3}$ axis. The octahedron formed by the $\mathrm{C}$ and $\mathrm{N}$ atoms of the coordination sphere is elongated by $3.73 \%$ along this $\mathrm{Fe} \bullet \bullet \mathrm{B}$ axis compared to a regular octahedron (mean distances). The Fe-N distances

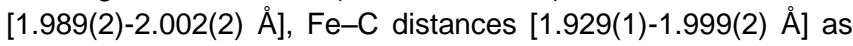
well as the effective magnetic moment at room temperature $\left(\mu_{\text {eff }}\right.$ $=2.26 \mu_{\mathrm{B}}$ ) unambiguously reveal a LS state for the $\mathrm{Fe}^{\text {III }}$ ion.

Due to the local triclinic symmetry of the $\mathrm{Fe}$ site, the six independent components $\chi_{11}, \chi_{22}, \chi_{33}, \chi_{23}, \chi_{31}$ and $\chi_{12}$ of the susceptibility tensor have to be refined. Therefore three PND data collections were performed at $2 \mathrm{~K}$ for three different orientations of the crystal, set along the vertical magnetic field. These directions, orthogonal to each other, were chosen with respect to the morphology of the single crystal (Fig. S1). The corresponding field components in the orthonormal basis $(\boldsymbol{i}, \boldsymbol{j}, \boldsymbol{k})$ related to the crystallographic axes $\left(\boldsymbol{a}^{*}, \boldsymbol{b}, \boldsymbol{c}\right)$ are reported in Table S4. The simultaneous refinement of the six components on the three sets of flipping ratios collected at $2 \mathrm{~K}$ leads to the following local susceptibility tensor:

$$
\overleftrightarrow{\chi}_{F e}=\left(\begin{array}{lll}
0.10(3) & 0.02(3) & 0.11(4) \\
0.02(3) & 0.27(7) & 0.16(2) \\
0.11(4) & 0.16(2) & 0.82(5)
\end{array}\right)\left[\operatorname{in} \mu_{B} / T\right]
$$

relatively to the orthonormal basis $(\boldsymbol{i}, \boldsymbol{j}, \boldsymbol{k})$.

The determination of the eigenvalues and eigenvectors (Table S5) associated with the tensor (2) leads to the susceptibility ellipsoid drawn in Fig. 1 (view along the $\mathrm{C}_{3}$ axis in Fig. S2). The shape of the ellipsoid is given by the relative strength of the eigenvalues, and its orientation is dictated by the eigenvectors $\left(\chi_{1}, \chi_{2}, \chi_{3}\right)$. It clearly shows the existence of a molecular easy magnetization direction in which the magnetic susceptibility $\left(\chi_{1}=\right.$
$0.87(7) \mu_{B}(T)$ is significantly larger than those in the two other principal directions $\left(\chi_{2}=0.23(8)\right.$ and $\left.\chi_{3}=0.09(5) \mu_{B} T\right)$.

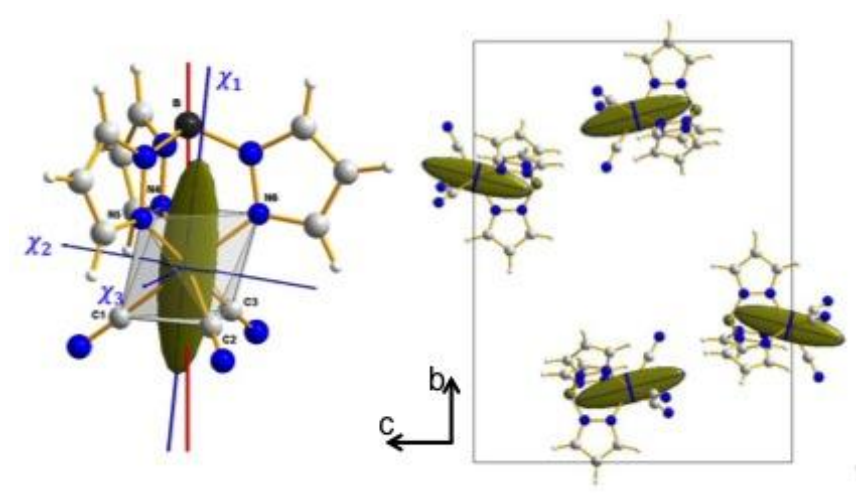

Figure 1. (left) Fe"II magnetic susceptibility ellipsoid obtained from PND within its coordination octahedron at $2 \mathrm{~K}$. The local easy magnetization axis ( $\chi_{1}$ in blue) is nearly parallel to the $\mathrm{C}_{3}$ pseudo-axis (in red) of the molecule. The overall size of the ellipsoid is set arbitrarily for a better encapsulation within the coordination octahedron. (right) Projection of the unit cell along the a-axis and representation of the magnetic susceptibility ellipsoids determined by PND for the four molecules at $2 \mathrm{~K}$.

The $\chi_{1}$ easy magnetization direction is found very close $\left(8^{\circ} \pm 5^{\circ}\right)$ to the $\mathrm{C}_{3}$ pseudo-axis (red solid line in Fig. 1). The two other magnetic principal directions $\chi_{2}$ and $\chi_{3}$ are almost contained in the plane parallel to the triangular faces N4-N5-N6 and C1-C2C3 of the octahedron. The local susceptibility ellipsoids on the four $\mathrm{Fe}$ sites in the unit cell are represented in Fig. 1 in projection along the a-axis. The molecular easy magnetization axes are mostly oriented along the $\mathbf{c}$-axis, whereas the $(\mathbf{a}, \mathbf{b})$ plane appears to be a crystallographic hard magnetization plane. For a quantitative comparison between PND and SQUID magnetometry, it is convenient to calculate the macroscopic susceptibility tensor of the crystal (associated with the unit cell) by summing the four local tensors determined by PND measurements. This leads to the crystal susceptibility tensor (3) expressed in the $(\boldsymbol{i}, \boldsymbol{j}, \boldsymbol{k})$ basis.

$\overleftrightarrow{\chi}_{\text {cell }}=\frac{1}{4} \sum_{n=1}^{4} \overleftrightarrow{\chi}_{n}=\left(\begin{array}{ccc}0.10(3) & 0 & 0.11(4) \\ 0 & 0.27(7) & 0 \\ 0.11(4) & 0 & 0.82(5)\end{array}\right)\left[\right.$ in $\left.\mu_{B} / T\right]$

The crystal magnetic principal directions and the associated eigenvalues $\left(\chi_{m 1}, \chi_{m 2}, \chi_{m 3}\right)$ are given in Table S6. In Eq. (3), and as expected for a monoclinic space group, the $\chi_{12}$ and $\chi_{23}$ components vanish when summing the four local tensors. Thus the $\mathbf{b}$-axis is necessarily a principal magnetic direction of the crystal $\left(\chi_{m 2}=\chi_{b}=0.27(7) \mu_{B} T\right)$. The two other magnetic principal directions $\chi_{m 1}$ and $\chi_{m 3}$ are then contained in the $(\mathbf{a}, \mathbf{c})$ plane. More precisely, the hard magnetization axis of the crystal $\chi_{m 1}=0.09(4) \mu_{B} T$ is found very close to the a-axis (within $2^{\circ}$ ) while the easy magnetization axis $\chi_{\mathrm{m} 3}=0.83(6) \mu_{\mathrm{B}} \mathrm{T}$ is perpendicular to it (Fig. S5). 


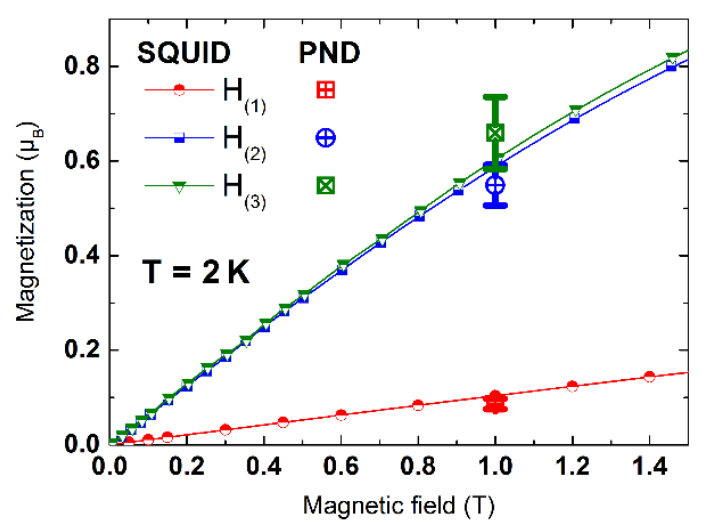

Figure 2. Magnetization values obtained from PND using the crystal susceptibility tensor (points with error bars for a field of $1 \mathrm{~T}$ ) compared to the magnetization curves from SQUID magnetometry at $2 \mathrm{~K}$, for three directions of the applied magnetic field in the range 0-1.5 $\mathrm{T}$ (continuous lines).

The fact that the hard magnetization axis of the crystal $\left(\chi_{m 1}\right)$ is found very close to the a-axis is perfectly compatible with the macroscopic SQUID magnetization data on single crystal (Fig. S3-S4). From the crystal susceptibility tensor (3), the magnetic susceptibilities can be calculated at $2 \mathrm{~K}$ for any direction of the applied magnetic field, allowing a quantitative comparison between PND and SQUID measurements. In the three directions $\mathrm{H}_{(1)}, \mathrm{H}_{(2)}$ and $\mathrm{H}_{(3)}$ (Fig. S1), the calculations lead to: $\chi_{(1)}=0.09$ (1), $\chi_{(2)}=0.55(4)$ and $\chi_{(3)}=0.66(8) \mu_{B} T$, in agreement with the values obtained by SQUID $\left(\chi_{(1)}=0.10, \chi_{(2)}=0.59\right.$ and $\chi_{(3)}=0.61$ $\mu_{B}(T)$. Although the magnetization error bars obtained by PND are relatively large, there is a good agreement for the three field orientations (Fig. 2). In practice, SQUID experiments have the advantage to measure the macroscopic magnetization in a given direction with a good accuracy, but very often the orientation of the single-crystal in the magnetic field is not perfectly known due to the manual mounting of the sample. The opposite situation is encountered with PND: the orientation of the single crystal under the external magnetic field is precisely known, since it is determined from orientation matrices, but the magnetic moments are affected by larger error bars.

EPR spectra performed on a powder sample of $\mathrm{PPh}_{4}\left[\mathrm{Fe}^{\text {III }}(\mathrm{Tp})(\mathrm{CN})_{3}\right] \cdot \mathrm{H}_{2} \mathrm{O}(\mathrm{T}=5-50 \mathrm{~K})$ are presented in Fig. 3. Three resonance peaks are observed at $\mathrm{H}_{(\mathrm{A})}=0.196 \mathrm{~T}, \mathrm{H}_{(\mathrm{B})}=$ $0.595 \mathrm{~T}$ and $\mathrm{H}_{(\mathrm{C})}=1.173 \mathrm{~T}$. The ground state of a LS Fe ${ }^{\mathrm{III}}$ ion $\left(d^{5}\right)$ in octahedral coordination is an orbital triplet with an unquenched first-order orbital momentum. However, additional low symmetry ligand field breaks the degeneracy of the orbital triplet term so that the ground state can be generally treated as a pure effective spin $S_{\text {eff }}=12$. The low temperature magnetic properties of the LS Fe"II ion can thus be described using the phenomenological Zeeman Hamiltonian:

$$
\widehat{H}^{\prime}=\mu_{B} \boldsymbol{S}_{\text {eff }} \cdot \stackrel{\leftrightarrow}{g}_{\text {eff }} \cdot \boldsymbol{H}
$$

where $\overleftrightarrow{g}_{\text {eff }}$ is the Landé tensor associated with the effective spin. This tensor incorporates the potential influence originating from the orbital contribution. The observed EPR transitions are explained considering an anisotropic Zeeman splitting of the ground Kramers doublet of the LS Fe"I, described by the
Hamiltonian (4). ${ }^{[17]}$ The three principal values of the effective Landé tensor can be deduced: $\mathrm{g}_{\mathrm{A}}=3.7, \mathrm{~g}_{\mathrm{B}}=1.1$ and $\mathrm{g}_{\mathrm{C}}=0.57$. This result is compatible with an axial type anisotropy with a pronounced molecular easy magnetization axis, in agreement with PND outcomes.

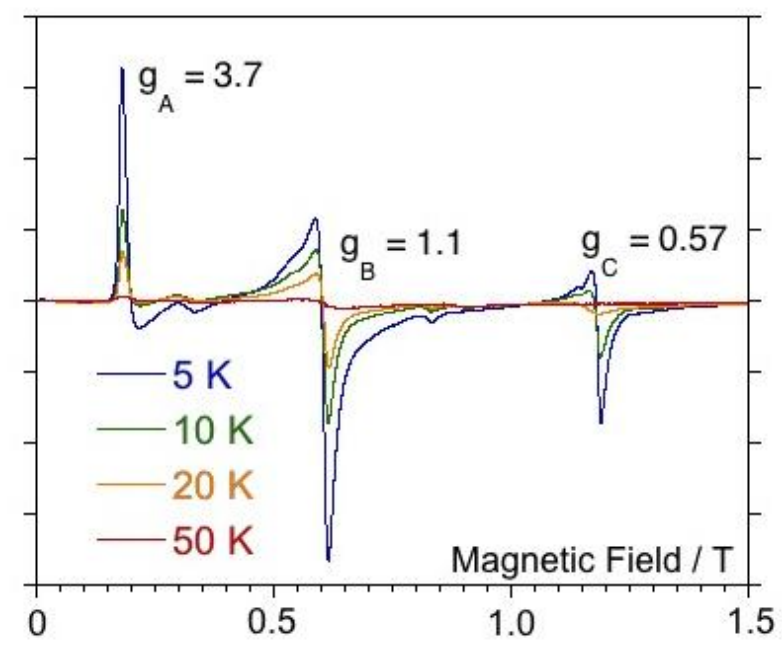

Figure 3. EPR spectra recorded at $9.42 \mathrm{GHz}$ (X-band) on a powder sample of $\mathrm{PPh}_{4}\left[\mathrm{Fe}\{\mathrm{Tp}\}(\mathrm{CN})_{3}\right] \cdot \mathrm{H}_{2} \mathrm{O}$ in the temperature range $[5-50 \mathrm{~K}]$.

To corroborate these experimental results, quantum chemical calculations were performed on the isolated $\left[\mathrm{Fe}^{\mathrm{III}}(\mathrm{Tp})(\mathrm{CN})_{3}\right]$ complex stemming from PND measurements and for which the position of hydrogen atoms were optimized at the DFT level (see computational details in SI). First, spin-orbit DFT calculations were carried out. As shown in Table S8, whatever the functional used, the calculated $g$-tensor remains strongly isotropic in contradiction with experimental observations. We thus moved to explicitly correlated $a b$ initio methods, i.e. SA-CASSCF/MSCASPT2 using three different sizes of active space CAS[n,m] (n electrons in $\mathrm{m}$ orbitals, Scheme S1). First, we considered a CAS[5,5] consisting of the five $3 d$ electrons of Fe $\mathrm{e}^{\text {III }}$ spanning the five $3 d$ orbitals. At the CASSCF level, the lowest state is the sextet (Fig. S6). However, a strong spin-orbit coupling is observed between the first two doublet excited states. Taking into account dynamical correlation through CASPT2 treatment allows stabilizing both the quartet and doublet states and thus recovering the correct energetic order with a fundamental doublet state. However, this is to the detriment of spin-orbit coupling between the different doublet states and as in DFT, the $g$-tensor is computed almost isotropic (Table S8). In order to improve the quality of the wavefunctions, the active space was enlarged to CAS[5,10] to take into account the so-called "double-shell effect". ${ }^{18]}$ At the CAS[5,10]PT2 level, the $g$-tensor shows large easy-axis anisotropy with $g_{x}=1.04, g_{y}=0.24$ and $g_{z}$ $=4.28$. Finally, two occupied $\sigma$-type orbitals that correspond to the lone pairs of the first coordination sphere nitrogen atoms were included in the active space, i.e. CAS[9,12]. The resulting improvement in the treatment of the electronic correlation allows obtaining a fundamental doublet already at the CASSCF level that is conserved at the PT2 level. A strong SO coupling is still 
operating resulting in a large $\mathrm{z}$-component of the g-tensor $\left(\mathrm{g}_{\mathrm{z}}=\right.$ 4.21) whereas $g_{x}$ and $g_{y}$ are 1.20 and 0.42 , respectively. The occupation of the molecular orbitals (Fig. S7) with the single electron perpendicular to the trigonal elongation of the $\mathrm{Fe}^{\mathrm{III}}$ coordination environment induces this strong SO coupling at the origin of the g-tensor anisotropy. The calculated $g$ components obtained at the best level of calculation, i.e. CAS[9,12]PT2, are in excellent agreement with the three principal values of the EPR effective Landé tensor (Fig. 3). Moreover, as shown in Fig. S8S9, they perfectly match the PND results with an easy magnetization direction found very close to the $\mathrm{C}_{3}$ pseudo-axis. Finally, the single crystal susceptibility and magnetization curves were reconstructed from the calculated data (Fig. S10). Here again, good agreement between theory and experimental magnetometry is obtained. The slight deviation with our experimental results may be due to the action of a pseudo JahnTeller effect but its role should be marginal. However, a proper treatment would necessitate a whole specific study based on quantum chemical approach coupled to ligand-field theory. ${ }^{[19]}$

In summary, we obtained a coherent picture of the magnetic anisotropy of the LS $\left[\mathrm{Fe}^{\mathrm{III}}(\mathrm{Tp})(\mathrm{CN})_{3}\right]^{-}$complex by PND, EPR, SQUID magnetometry and quantum chemistry calculations. The method used to interpret the PND data allows a precise determination of the local magnetic susceptibility tensor on each individual magnetic site in the unit cell. It clearly reveals (a) the existence of a local easy magnetization axis with one principal value unequivocally larger than the two others $\chi_{1}>\chi_{2}$ and $\chi_{3}$ and (b) a straightforward correlation between this magnetic principal direction $\left(\chi_{1}\right)$ with the $\mathrm{C}_{3}$ molecular pseudo-axis. From the PND results, we show that the magnetic anisotropy is driven by the trigonal elongation direction of the coordination octahedron, giving rise to molecular easy magnetization axis, as predicted by high-level quantum chemical calculations. Through this simple example, we demonstrate that one can access the local magnetic principal directions, as well as the associated susceptibility values, regardless of the complexity of the crystal structure. This work opens new perspectives in the field of molecular magnetism for a better understanding of magnetic anisotropy and, in particular, with the aim of determining the structural and chemical characteristics, which induce a strong anisotropy. This procedure may also be advantageously applied in polynuclear complexes in order to determine the independent local susceptibility tensor on each magnetic center in the molecule.

\section{Acknowledgements}

Computations were performed using HPC resources from GENCI-CINES/IDRIS (Grant 2014-80649). KC and CB thanks the ANR for financial support.

Keywords: molecular magnetism - magnetic anisotropy • polarized neutron diffraction $\cdot$ ab intio calculations $\cdot$ EPR
[2] (a) J. Stöhr, H. A. Padmore, S. Anders, T. Stammler, M. R. Scheinfein Surf. Rev. Lett. 1998, 5, 1297-1308; (b) R. Sessoli, M. Mannini, F. Plneider, A. Cornia, P. Sainctavit, in Magnetism And Synchrotron Radiation: New Trends (Eds.: E. Beaurepaire, H. Bulou, R. Scheurer, J.P. Kappler), Springer Proceeding in Physics, 2010, 133, pp. 279-311.

[3] F.H. Köhler, in Magnetism: Molecule to Materials (Eds.: J.S Miller, M. Drillon), Wiley-VCH, Weinheim, 2001, pp. 379-340.

[4] (a) A. Zheludev, A. Grand, E. Ressouche, J. Schweizer, B. G. Morin, A. J. Epstein, D. A. Dixon, J. S. Miller, Angew. Chem. Int. Ed. 1994, 33, 1397-1399; (b) E. Ruiz, G. Rajamaran, S. Alvarez, B. Gillon, J. Stride, R. Clérac, J. Larionova, S. Decurtins, Angew. Chem. Int. Ed. 2005, 44, 2711-2715.

[5] D. Gatteschi, R. Sessoli, J. Villain, in Molecular Nanomagnets, Oxford University Press, New York, 2006, pp 108-156.

[6] (a) W. T. Cong, Z. Tang, X. G. Zhao, J.H. Chu, Nat. Commun. 2015 DOI: 10.1038/srep09361; (b) A. Candini, S. Klyatskaya, M. Ruben, W. Wernsdorfer, M. Affronte, Nanoletters 2011, 11, 2634-2639; (c) P. Gambardella, S. Stepanow, A. Dmitriev, J. Honolka, F. M. de Groot, M. Lingenfelder, S. Sen Gupta, D. D. Sarma, P. Bencok, S. Stanescu, S. Clair, S. Pons, N. Lin, A. P. Seitsonen, H. Brune, J. V. Barth, K. Kern, Nature Mat. 2009, 8, 189-193.

[7] (a) P. J. Ferko, S. M. Holmes, Cur. Inorg. Chem. 2013, 3, 172-193; (b) S. Wanga, X.-H. Dinga, J.-L. Zuob, X.-Z. Youb, W. Huanga, Coord Chem. Rev. 2011, 255, 1713-1732.

[8] see for example: (a) B. Figgis, E. S. Kucharrski, M. Vrtis, J. Am. Chem. Soc. 1993, 115, 176-181; (b) Y. Pontillon, A. Bencini, A. Caneschi, A. Dei, D. Gatteschi, B. Gillon, C. Sangregorio, J. Stride, F. Totti, Angew. Chem. Int. Ed. 2000, 39, 1787-1788.

P. J. Brown, J. Phys. Chem. Solid 2004, 65, 1977-1983.

[10] A. Gukasov, P. J. Brown, J. Phys.: Condens. Matter 2002, 14, 88318839.

[11] A. Gukasov, P. Rogl, P. J. Brown, M. Mihalik, A. Menovsky, J. Phys. Condens. Matter 2002, 14, 8841-8851.

[12] A. Gukasov, H. Cao, I. Mirebeau, P. Bonville, Physica B 2009, 404, 2509-2512.

[13] A. Borta, B. Gillon, A. Gukasov, A. Cousson, D. Luneau, E. Jeanneau, I. Ciumacov, H. Sakiyama, K. Tone, M. Mikuriya, Phys. Rev. B 2011, 83, 184429-1-5.

[14] O. Zaharko, M. Pregelj, A. Zorko, R. Podgajny, A. Gukasov, J. Van Tol, S. I. Klokishner, S. Ostrovsky, B. Delley, Phys. Rev. B 2013, 87, 024406-1-10.

[15] R. Lescouëzec, J. Vaissermann, F. Lloret, M. Julve, M. Verdaguer, Inorg. Chem. 2002, 41, 5943-5945.

[16] See for example : M. Perfetti, L. Lucaccini, L. Sorace, J.-P. Costes, R. Sessoli, Inorg. Chem. 2015, 53, 3090-3092.

[17] B. Bleaney, M. C. O'Brien, Proc. Phys. Soc. 1956, 69, 1216-1230.

[18] M. Kepenekian, V. Robert, B. Le Guennic, C. de Graaf, J. Comput. Chem. 2009, 30, 2327-2333 and references therein.

[19] (a) M. Atanasov, P. Comba, C. A. Daul, A. Hauser, J. Phys. Chem. A 2007, 111, 9145-9163. (b) Atanasov et al. Inorg. Chem. 2011, 50, 7460; (c) Peric et al. Chem. Eur. J. 2015, 21, 3716 


\section{COMMUNICATION}

The method of the local site susceptibility tensor applied to PND measurements allows to probe the magnetic properties at the atomic scale and to draw a clear picture of the magnetic anisotropy in molecular complexes such as the $\left[\mathrm{Fe}^{\text {III }}(\mathrm{Tp})(\mathrm{CN})_{3}\right]^{]}$.

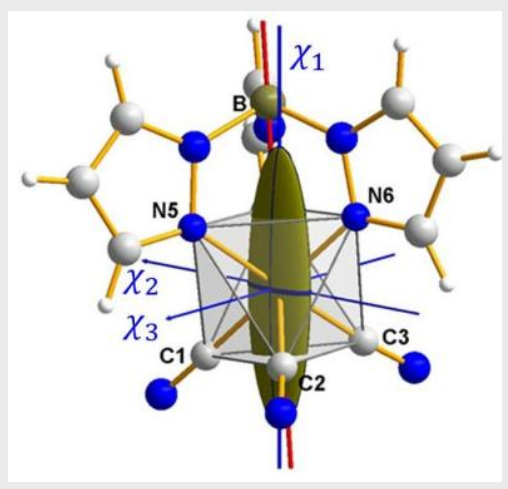

Karl Ridier, ${ }^{[a]}$ Abhishake Mondal, ${ }^{[b]}$ Corentin Boilleau, ${ }^{[c]}$ Olivier Cador, ${ }^{[c]}$ Béatrice Gillon, ${ }^{* a]}$ Grégory Chaboussant, ${ }^{[a]}$ Boris Le Guennic ${ }^{*},{ }^{[c]}$ Karine Costuas, ${ }^{[c]}$ Rodrigue Lescouëzec ${ }^{\star[b]}$

Page No. - Page No.

A direct picture of the local magnetic anisotropy by polarized neutron diffraction (PND) confronted to EPR, magnetometry and ab initio investigations 